Relations industrielles

Industrial Relations

\title{
L'avenir de la Loi de la convention collective
}

Volume 4, numéro 4, décembre 1948

URI : https://id.erudit.org/iderudit/1023454ar

DOI : https://doi.org/10.7202/1023454ar

Aller au sommaire du numéro

Éditeur(s)

Département des relations industrielles de l’Université Laval

\section{ISSN}

0034-379X (imprimé)

1703-8138 (numérique)

Découvrir la revue

Citer cet article

(1948). L'avenir de la Loi de la convention collective. Relations industrielles / Industrial Relations, 4(4), 34-36. https://doi.org/10.7202/1023454ar

Tous droits réservés (C Département des relations industrielles de l’Université Laval, 1948
Ce document est protégé par la loi sur le droit d'auteur. L’utilisation des services d'Érudit (y compris la reproduction) est assujettie à sa politique d'utilisation que vous pouvez consulter en ligne.

https://apropos.erudit.org/fr/usagers/politique-dutilisation/ 


\section{L'AVENIR DE LA LOI DE LA CONVENTION COLLECTIVE}

\author{
Une opinion sur le rôle de cette loi \\ et les améliorations projetées
}

Plusieurs fois, dans le passé, le Bulletin des relations industrielles, a traité de la Loi de la convention collective et a exposé ses origines, ses principes et ses réalisations.

Aujourd'hui, je voud̄rais considérer son avenir et, pour cela, exposer le rôle particulier qu'elle poursuit et les amendements projetés.

$\mathrm{Au}$ cours des dernières anmées, on a amendé la Loi du salaire minimum et l'on a introduit, dans les Statuts, la Loi des relations ouvrières. Tout cela, c'est très bien, pourvu que la mise en vigueur de nouvelles mesures ne place pas, sur un degré d'infériorité, une loi qui doit rester au premier plan de notre législation à cause de la valeur de ses principes, et à cause du souci de bonne entente, de coopération et de paix sociale qui en constitue le fondement.

Mais, pour bien comprendre la portée des amendements projetés, il faut, en premier lieu, se rappeler le rôle particulier que la Loi de la convention collective a joué depuis 1934 et celui qu'elle est appelée à remplir dans l'avenir.

\section{I-Rôle propre à cette loi}

Dès sa mise en vigueur, en 1934, la Loi de la convention collective a démontré être la mesure la plus efficace pour assurer le relèvement des conditions de travail des employés de la province, pendant les années de dépression. Elle était, en effet, la seule loi capable de surmonter le grand obstacle à l'amélioration des salaires, à savoir, la concurrence entre employeurs sur le gagne-pain ou les gages du travailleur. Elle faisait disparaitre cette concurrence en généralisant ou en imposant, à toute une industrie, les salaires, les heures de travail et les conditions d'apprentissage contenus dans les clauses d'un contrat passé par un groupe de patrons et d'ouvriers de cette industrie.

Aujourd'hui, cette concurrence sur les salaires étant moins forte, on sent moins le besoin, en

N.B.-Cet article nous a été adressé par une personne qui possède une grande expérience dans l'application de notre législation du travail. Nous croyons qu'il soulève des points très importants et nous invitons les lecteurs qui le jugent à propos de nous communiquer leurs commentaires. certains milieux, de recourir à la convention collective généralisée. Advienne, toutefois, une période de dépression où la concurrence sur les salaires fera de nouveau loi, si la convention collective généralisée n'existe plus, les travailleurs et les employeurs tomberont dans le même état de concurrence et de déchéance dont ils souffraient en 1933.

Je n'insisterai pas sur le fait que la Loi de la convention collective compte environ 97 décrets dans la province et régit les salaires de près de 225,000 ouvriers; ce n'est là qu'un fait, et non une caractéristique spéciale à cette loi. Par contre, on ne saurait trop appuyer sur les points suivants :

1) Aucune législation ne saurait apporter une plus grande protection aux salariés, du fait que la convention, avec extension juridique, couvre tous les ouvriers d'une industrie sans distinction, que le comité paritaire, chargé de l'application du décret, a le pouvoir de vérifier la liste de paie, d'exiger des rapports de l'employeur, de demander, sous serment au besoin, des renseignements à l'employeur et à l'employé, de réclamer en justice en faveur des salariés, sans demander leur autorisation et mème s'ils renoncent à leurs droits. Aucune mesure ne pourrait donner davantage, ou même s'en approcher.

2) Les comités paritaires ne se sont pas bornés aux intérêts matériels des membres, mais se sont aussi occupés des intérêts intellectuels des employeurs et des employés de l'industrie. Ce son' les comités paritaires qui ont formé, avec l'aide du ministère du Travail, les commissions d'apprentissage, organisées en vertu de la Loi de l'aide à lapprentissage; et je ne crois pas me tromper en affirmant que, sans les comités paritaires, il n'existerait peut-être pas une seule commission d'apprentissage dans la province. Ces commissions d'apprentissage obtiennent aujourd'hui des résultats inespérés du fait qu'elles parviennent à former des employés compétents, pour l'industrie, telle qu'elle existe en 1948, qu'elles contribuent à hausser le niveau intellectuel des travailleurs, et qu'elles mettent en application, chez nous, ce principe qui veut que la formation professionnelle des employés appartienne, en premier lieu, à lindustrie, avant de tomber dans les mains de l'Etat.

3) Signalons, en plus, que là où une convention collective généralisée est mise en vigueur, l'industrie peut difficilement être dominée ou per- 
sécutée par une organisation dirigée par des chefs communistes. Le comité paritaire, en effet, est composé des representants de différentes associations ouvrières, en plus des représentants des associations patronales. Former un comité paritaire, c'est soustraire, dans presque tous les cas, une industrie à l'influence communiste. Et ici, il est sans doute bon de rappeler que, l'an dernier, sans l'intervention opportune et énergique d'un comité paritaire, aidé par les organisations ouvrières et patronales, une de nos plus belles industries, qui compte plus de $98 \%$ de catholiques, serait aujourd'hui dirigée par la main sordide d'un chef communiste.

\section{II -- Les modifications suggérées}

Les quelques faits mentionnés plus haut prouvent que la Loi de la convention collective joue un rôle qui lui est propre et semblent suffisants pour démontrer qu'il est absolument important de donner à cette loi l'attention la plus soigneuse. $\mathrm{Si}$ l'on veut qu'elle continue le rôle qu'elle a rempli jusqu'à date, il importe d'étudier avec grande bienveillance, les amendements proposés en vue de lui conserver son rang et son prestige.

Il y a lieu, tout d'abord, de porter attention à toutes les suggestions d'amendements qui visent à faciliter la mise en application ou le respect intégral des décrets. Je mentionnerai, entre autres, les recommandations de bien définir les termes « employeur et employeur professionnel»; de donner aux comités paritaires le pouvoir d'exercer les recours qui naissent de la loi, d'exiger un système d'enregistrement de travail et des rapports de toute personne assujettie au décret; de s'assurer que les heures de travail comprennent le temps ou l'employé est tenu de rester à la disposition de l'employeur; de statuer que le décret a priorité sur les règlements municipaux en ce qui a trait aux heures d'ouverture et de fermeture, et a préséance sur les conventions passées en vertu de la Loi des syndicats professionnels; d'arrêter le cours de la prescription par l'envoi de lettres enregistrées; et, en général, de considérer tout amendement qui, se basant sur l'expérience, tend à fermer la brèche partout où les adversaires ont réussi à attaquer avec succès.

Il importe, en second lieu, d'insérer dans cette loi, les amendements qui la maintiendront au premier rang des lois sociales dans l'esprit des employeurs et employés de la province. La loi actuelle est limitée, notamment, aux salaires, aux heures de travail et à l'apprentissage. Son champ d'action ne pourrait-il pas être plus étendu, de façon à couvrir tout le domaine de la sécurité sociale? De ce fait, la convention pourrait contenir, par exemple, des clauses relatives au fonds de pension, au bénéfice-décès, à l'assurance-maladie et à tout service similaire. ${ }^{1}$

$\mathrm{Ne}$ pourrait-elle pas, en plus être amendée de façon que les organisations ouvrières et patronales aient autant d'intérêt à négocier en vue d'une convention collective, avec extension juridique, qu'en vue de toute autre loi ?

Soyons précis. Si, pour ne citer qu'un exemple, d'autres lois permettent des clauses de sécurité syndicale, et si la Loi de la convention collective ne les permet pas, les organisations ouvrières adopteront les autres lois et abandonneront la Loi de la convention collective. Si l'on veut que les parties contractantes soient intéressées à signer des conventions, en vertu de cette loi, il faut s'assurer que les organisations y trouveront l'intérêt de l'association en même temps que l'intérêt des membres.

Je sais que l'on porte objection à cette affirmation en distinguant entre les clauses normatives et les clauses contractuelles et en affirmant que les clauses normatives peuvent être généralisées tandis que les clauses contractuelles ne devraient pas l'être.

Tout en admettant la valeur et l'à-propos de cette distinction, je resie d'opinion que de telies

(1) N.D.L.R.-Dans le même ordre d'idées récemment, John L. Lewis exprimait dans U.S. News \& World Report, 19 novembre 1948, les raisons pour lesquelles la sécurité sociale devrait d'abord être l'affaire de l'industrie avant celle du gouvernement: "There's a definite principle involved of making the commodity, used generally by the public or special interests, bear completely its own cost of production, rather than have the production of that commodity subsidized to some degree either by the Government or by tax-collection processes of Government. We hold that the care of the human element in an industry should inherently run with the cost of production. A man is just as essential as any other item in the cost of production... We hold that in order to maintain our system of free enterprise we shouldn't expect the taxpayers of the country to assume the burden of caring for the human wastage in an especially hazardous or bad industry like the mining industry; that the nonconsumer of coal should not be taxed to keep up the plant and equipment of the coal company. And we hold that part of the plant and equipment is the man power and personnel... We hold that the proper care of the human element in the mining industry or any other major industry should properly be charged to the cost of production and not assessed against the taxpayers as a whole. To that degree, we think it's a step in the direction of free government. It's contrary to the concept that the Government should do everything for its citizens. The industry should do it, and the commodity should bear the cost of it - whatever that may be. This is a chance for labor and management to take care of these problems and eliminate the necessity for the Government to build up huge, inefficient and costly administrative bureaus to try to do the task in a less efficient way. To me it's an assurance of the preservation of free enterprise." 
restrictions paraissent aller à l'encontre de la lettre même de la loi qui spécifie que peuvent être généralisées toutes les clauses d'un contrat jugées par le Lieutenant-Gouverneur en Conseil conformes à l'esprit de la loi. Lorsque cet article a été inséré dans la loi, il avait pour but de permettre l'extension de toutes les clauses d'une convention collective qui pouvait convenir à un commerce ou à une industrie. Ce n'est qu'à la suite d'opinions légales, qu'on est venu à donner à cette clause la portée restrictive qu'on lui connaît présentement.

Ce qu'il importe, aujourd'hui, ce n'est pas de faire valoir des raisons de limiter le champ d'action de la loi; mais, d'étudier, de chercher et de trouver le moyen de l'étendre à tout le domaine et à tous les problèmes des relations patronalesouvrières.

Il faudrait, en troisième lieu, que les mêmes facilités soient accordées à ceux qui veulent négocier en vertu de la Loi de la convention collective qu'à ceux qui veulent négocier en vertu de la Loi des relations ouvrières. Il faudrait, en d'autres mots, que l'on assure à ces parties contractantes, non seulement la conciliation et l'arbitrage pour la négociation des contrats, mais encore, lorsque le décret est en vigueur, que les différends puissent être soumis à un tribunal de travail ca- pable de régler les problèmes de façon expéditive, sans passer par toutes les complications et toutes les longueurs de nos cours de justice.

En résumé, si l'on veut que la Loi de la convention collective continue à remplir son ròle, si l'on veut qu'elle ne dépérisse pas, mais progresse, il faut qu'elle suive l'évolution; qu'elle soit renforcée partout où ses adversaires trouvent des points faibles; qu'elle soit amendée d'après les données de l'expérience; que les parties contractantes trouvent intérêt à négocier en vertu de cette loi de préférence à toute autre; qu'elle bénéficie de tous les avantages des lois existantes aussi bien pour la négociation des décrets que pour leur mise en application.

Le Bulletin des relations industrielles diffuse des principes et des faits. Rien ne l'empêche, à mon sens, de soumettre aussi des opinions ou des sujets d'étude. Il se peut que je fasse erreur, mais je crains que si la Loi de la convention collective est restreinte en des cadres trop étroits pour répondre aux besoins actuels, elle sera délaissee malgré tous les services qu'elle a pu rendre et qu'elle rend présentement. Cette loi a été édifiée sur un principe merveilleux; il s'agit de lui donner des possibilités d'agir.

\title{
MOUVEMENT DES SALAIRES MINIMA DANS L'INDUSTRIE DU BÂTIMENT
}

\author{
GÉrard RoY
}

Dans la plupart des régions de la province, les conditions de travail des ouvriers des métiers de la construction sont maintenant réglementées par des décrets pris en exécution de la Loi de la convention collective. Après 15 ans d'application de la Loi dans ce domaine, il est très intéressant d'analyser les 84 décrets de base passés depuis 1934 et les 335 arrêtés ministériels les amendant, en vue d'en tirer des statistiques très utiles aux associations patronales et ouvrières, aux comités paritaires chargés de surveiller et d'assurer l'observance des décrets et à tous ceux qui s'intéressent aux problèmes des relations industrielles.

Les tableaux suivants concernant les métiers de charpentier-menuisier, d'électricien et de briqueteur, donnent une idée générale des salaires minima fixés par les décrets dans les principales régions depuis 1934 .

Selon l'Office fédéral de la Statistique, l'indice du coût de la vie le plus récent, novembre 1948, est de 159.6. La comparaison entre l'augmentation du coût de la vie et la hausse des salaires peut être faite dans chaque région; cependant, avant de tirer des conclusions définitives sur ces phénomènes, il faut tenir compte de quelques facteurs: niveau réel du coût de la vie, salaires qui n'étaient pas assez élevés en 1939, puissance des organisations professionnelles, etc.

Il est très important de souligner le fait que les salaires minima rendus obligatoires par décrets sont une garantie pour les ouvriers et les patrons, n'étant pas sujets aux fluctuations que peut provo- 